\title{
LA DINÁMICA DEL SALUDO EN INTERACGIONES INSTITUCIONALES EN ESPAÑOL BONAERENSE
}

\author{
Gisele G. JuliáN \\ Centro de estudios lingüisticos «Dra. M. B. Fontanella de Weinberg». \\ Dpto. Humanidades, Universidad Nacional del Sur (UNS), \\ Bahía Blanca, Argentina / CONICET
}

\section{RESUMEN}

Entre los fenómenos dinamizantes de la (des)cortesía verbal los saludos resultan de particular interés en las interacciones institucionales, tanto desde el punto de vista de su expresión como de su percepción (Placencia 2001; Ferrer 2003; Rigatuso 2003 y 2015; Sánchez Lanza 2003; Murillo Medrano 2004). En este trabajo, que forma parte de una investigación sobre la expresión y percepción de la (des)cortesía en puestos de atención al público de instituciones del dominio de la salud y la educación en una cuidad perteneciente a la región lingüística del español bonaerense, proponemos analizar la dinámica del saludo en las interacciones desarrolladas entre empleados y usuarios en instituciones del dominio educativo, considerando las instancias de apertura y de cierre del hecho comunicativo. El estudio de este fenómeno adquiere relevancia ya que los saludos constituyen actos de habla destacados por los usuarios como fundamentales para la expresión de cortesía en dichos contextos. En el análisis atendemos en particular a la presencia/ausencia de saludos, los esquemas en los que se insertan, las fórmulas utilizadas, quién los formula y en qué medida contribuyen a la expresión de cortesía en el discurso institucional.

La investigación se enmarca en un enfoque de Sociolingüística interaccional (Gumperz 1982 y 2001; Tannen 1985, 1996 y 2004), con aportaciones de Etnografía de la comunicación (Gumperz y Hymes 1972), Microsociología de Goffman (1967) y Análisis del discurso (Drew y Sorjonen 2000). Para el estudio de los fenómenos de cortesía seguimos la Pragmática sociocultural (Bravo 1999; Bravo y Briz 2004; Kaul 2008 y 2010).

PALABRAS CLAVE: (des)cortesía verbal, español bonaerense, interacción institucional, saludo.

\section{ABSTRACT}

Among verbal (im) politeness phenomena, greetings are particularly important in institutional interactions, both from the point of view of their expression and their perception (Placencia 2001; Ferrer 2003; Rigatuso 2003 and 2015; 
Sánchez Lanza 2003; Murillo Medrano 2004). In this paper, which is part of a broader research on expression and perception of (im) politeness in customer service encounters in health and educational institutions in a city in the linguistic area of the Buenos Aires Spanish variety, we propose to analyze the dynamics of greetings in interactions between employees and users in educational institutions, specifically considering the opening and closing instances of the communicative event. Greetings, as speech acts, are emphasized by the users as fundamental for the expression of politeness in these contexts. We attend in particular to the presence/absence of greetings, the schemes in which they are inserted, the formulas used, who formulates them and how they contribute to the expression of politeness in the institutional discourse.

Our theoretical and methodological approach is based on Interactional Sociolinguistics (Gumperz 1982 and 2001; Tannen 1985, 1996 and 2004), with contributions from Ethnography of communication (Gumperz and Hymes 1972), Goffman's Microsociology (1967) and Discourse analysis (Drew and Sorjonen 2000). For the study of politeness phenomena, we follow leading researchers in Sociocultural Pragmatics (Bravo 1999; Bravo and Briz 2004; Kaul 2008 and 2010).

KEYWORDS: Buenos Aires Spanish Variety, greetings, (im)politeness, institutional interaction.

\section{INTRODUCCIÓN}

En las últimas décadas, siguiendo los lineamientos de la Sociolingüística interaccional (Gumperz 1982 y 2001; Tannen 1985, 1996 y 2004), el Análisis del discurso institucional (Drew y Sorjonen 2000), la Sociopragmática (Spencer-Oatey 2008) y la Pragmática sociocultural (Bravo 2009; Bravo y Briz 2004), han tenido un desarrollo destacado las investigaciones sobre interacción verbal en dominios institucionales. En ese marco, ha adquirido especial interés la problemática de la producción de discursos de (des)cortesía en puestos de atención al público de carácter institucional en vinculación con las percepciones de los hablantes acerca de los usos registrados (por ejemplo, Placencia 2001; Murillo Medrano 2004).

Entre los fenómenos dinamizantes de la (des)cortesía verbal los saludos resultan de particular relevancia en las interacciones institucionales. Por tal motivo, muchas investigaciones sobre (des)cortesía en dominios institucionales han dedicado especial atención al abordaje de este acto de habla, tanto desde el punto de vista de su expresión como de su percepción (Placencia 2001; Ferrer 2003; Rigatuso 2003 y 2015; Sánchez Lanza 2003; Murillo Medrano 2004). Dentro de esta línea, el artículo propone el abordaje de la dinámica del saludo en puestos de atención al público en inter- 
acciones institucionales en español bonaerense, fenómeno destacado especialmente por los usuarios como expresión de cortesía en esos dominios.

Este trabajo forma parte de una investigación ${ }^{1}$ más amplia sobre la expresión y percepción de la (des)cortesía en las interacciones entre empleados y usuarios que tienen lugar en puestos de atención al público de instituciones del dominio de la salud y la educación en una variedad dialectal del español de la Argentina. La comunidad objeto de estudio es una ciudad perteneciente a la región lingüística del español bonaerense. Nuestro propósito en este artículo es examinar la dinámica de los saludos en las interacciones desarrolladas entre empleados y usuarios en instituciones del dominio educativo, considerando las instancias de apertura y de cierre del hecho comunicativo. El estudio de este fenómeno adquiere relevancia fundamental en los intercambios comunicativos que tienen lugar en el conjunto de instituciones que nos ocupan, ya que los saludos constituyen actos de habla que, tal como relevamos a partir de la aplicación de tests de hábitos sociales en investigaciones previas (Julián 2015), son destacados por los usuarios como esenciales para la expresión de cortesía en dichos contextos. En el análisis atendemos en particular a la presencia/ausencia de saludos, los esquemas en los que se insertan, las fórmulas utilizadas, quién los formula y en qué medida contribuyen a la expresión de cortesía en el discurso institucional.

La investigación se enmarca en un enfoque de Sociolingüística interaccional (Gumperz 1982 y 2001; Tannen 1985, 1996 y 2004), con aportaciones de Etnografía de la comunicación (Gumperz y Hymes 1972), Microsociología de Goffman (1967) y Análisis del discurso (Drew y Sorjonen 2000). Para el estudio de los fenómenos de cortesía seguimos la Pragmática sociocultural (Bravo 1999; Placencia 2001 y 2004; Bravo y Briz 2004; Kaul 2008). Integramos además, en relación con el objeto de estudio de este trabajo, los postulados de Goffman (1955), Placencia (2001 y 2004), Ferrer y Sánchez Lanza (2002), Areiza Londoño (2003 y 2005), Ferrer (2003), Sánchez Lanza (2003), Murillo Medrano (2004) y Rigatuso (2003, 2008 y 2015). Finalmente, en el análisis seguimos el modelo de estructura conversacional que aplica Rigatuso en sus estudios sobre la dinámica interaccional de las fórmulas de tratamiento en el español bonaerense en la interacción comercial de la variedad (por ejemplo 1987, 2000, 2015), elaborado sobre

${ }^{1}$ La investigación, correspondiente a una tesis de Doctorado en Letras con orientación en Lingüística de la UNS, contó con el apoyo de sucesivas Becas Internas de CONICET desarrolladas bajo la dirección de la Dra. Elizabeth M. Rigatuso, y se inserta en un Proyecto de grupo de investigación dirigido por la Dra. Rigatuso («Estilo(s) comunicativo(s) y variación pragmática en la interacción verbal del español bonaerense: construcción de identidades, valores y creencias», subsidiado por la Secretaría de Ciencia y Tecnología de la Universidad Nacional del Sur). 
pautas básicas de organización interactiva del discurso: la estructura postulada por Teun van Dijk (1983: 276-280).

A continuación presentamos las consideraciones metodológicas de esta investigación, seguidas de algunas aproximaciones teóricas sobre el acto de habla saludo. En primer lugar abordamos el análisis de los saludos en la instancia de apertura del hecho comunicativo en las interacciones desarrolladas en las instituciones del dominio educativo objeto de estudio, y, en segundo lugar, examinamos los saludos en la instancia de cierre de la interacción en esos dominios. Finalmente, el último apartado presenta las conclusiones a las que arribamos a partir del análisis.

\section{CONSIDERACiOnes Metodológicas}

De acuerdo con el marco teórico señalado, la investigación se realiza siguiendo los lineamientos metodológicos de la Sociolingüística (Labov 1970; Moreno Fernández 1990; López Morales 1994; Hernández Campoy y Almeida 2005), en particular en su vertiente de Sociolingüística interaccional, en la propuesta de Gumperz (1982a y b y 2001), de la Antropología lingüística (Duranti 2000) y de la Pragmática sociocultural (Bravo 1999 y 2009; Bravo y Briz 2004).

Dentro del conjunto amplio y variado de los puestos de atención al público hemos realizado una selección, orientada por observaciones previas efectuadas como miembro de la comunidad, de acuerdo con el interés y la complejidad advertidos en su dinámica. Tales puestos corresponden a una institución educativa pública y una privada, ambas de nivel superior, y una institución del ámbito educativo en la que los docentes realizan diversos trámites relacionados con la labor docente. En el caso de la institución educativa pública se escogieron, a su vez, dos puestos de atención que corresponden, por un lado, a aquellas ventanillas y escritorios de una dependencia dedicada a la atención de trámites de alumnos de la institución y, por otro, a puestos de un Departamento Académico de la Universidad -el equivalente a una facultad en la estructura universitaria- donde se atiende tanto a alumnos como a docentes. En relación a la institución privada, hemos analizado las interacciones desarrolladas en el único puesto de atención al público que posee, una ventanilla de la oficina administrativa en la que atienden a docentes y alumnos actuales o potenciales. Por otra parte, como ya señalamos, hemos seleccionado una institución educativa pública a la que concurren docentes y estudiantes de carreras vinculadas a la docencia de todos los niveles para realizar diversos trámites y consultas relacionados con dicha labor. En este caso, hemos escogido puestos de atención al público de dos oficinas en las que los empleados atienden 
en escritorios ${ }^{2}$. La mayoría de las personas encargadas de la atención al público en estos dominios son mujeres.

Para la conformación del corpus se empleó una combinación de técnicas de trabajo de campo de carácter etnográfico orientadas a relevar uso y percepción. En relación con el uso concreto, aplicamos la técnica de participante-observador, mientras que para relevar la percepción de los usuarios aplicamos tests de hábitos sociales y realizamos experiencias con usuarios-jueces que actúan como evaluadores de algunas interacciones. En este trabajo abordamos concretamente el uso relativo a un acto de habla específico, el saludo, aunque hacemos algunas referencias a las percepciones de los usuarios cuando el análisis lo requiere.

Como hemos anticipado, con el fin de relevar el uso concreto utilizamos la técnica de participante-observador (Labov 1970; Gumperz 1982) en sus distintos modos de realización: participante-observador y observador no participante (Moreno Fernández 1990). En el primer caso, registramos interacciones naturales producidas en los contextos estudiados en las que la investigadora participa activamente. En el caso de observador no participante, la aplicación de la técnica consiste en acompañar durante la consulta o el trámite a usuarios que nos prestan su colaboración y en la toma de datos de interacciones producidas en esos dominios. La mayoría de las interacciones han sido grabadas, manteniendo el anonimato de los participantes como parte del compromiso ético de este tipo de investigaciones (Gabbiani y Madfes 2006), y luego transcriptas siguiendo las convenciones de transcripción incluidas en van Dijk (2000) e ideadas por Gail Jefferson $^{3}$. Además se tomaron notas etnográficas (Duranti 2000).

${ }^{2}$ En la descripción de los puestos de atención al público en los que se desarrollan las interacciones objeto de análisis brindamos información que consideramos relevante evitando, sin embargo, revelar datos que impidieran mantener el anonimato de las instituciones.

\begin{tabular}{|c|c|}
\hline$[$ ] & Simultaneidad de emisiones o fragmentos de emisiones. \\
\hline$::::$ & Alargamiento de la sílaba previa. \\
\hline MAYÚSCULAS: & Mayor volumen de emisión o fragmentos de emisión. \\
\hline${ }^{\circ}$ Símbolos de $\operatorname{grado}{ }^{\circ}:$ & Suavidad o amplitud disminuida de emisiones incluidas. \\
\hline Subrayado: & Énfasis mayor en la emisión del hablante. \\
\hline Signo igual $=$ & $\begin{array}{l}\text { No transcurrió tiempo entre los objetos conectados } \\
\text { por el signo. Significa que el hablante siguiente inicia } \\
\text { su emisión al final de la emisión del hablante actual. }\end{array}$ \\
\hline Punto entre paréntesis (.): & Pausa de una décima de segundo. \\
\hline Números entre paréntesis (1.7): & $\begin{array}{l}\text { Segundos y décimas de segundos entre turnos de los } \\
\text { hablantes o dentro de un mismo turno. }\end{array}$ \\
\hline ?: & Entonación interrogativa. \\
\hline ((Doble paréntesis)): & Aclaraciones, descripciones. \\
\hline Paréntesis incluyendo una $\mathrm{x}$ : (x) & Detención repentina o vacilación por parte del hablante. \\
\hline
\end{tabular}


Se registraron 150 interacciones en las instituciones del dominio educativo entre los años 2011 y 2015, lo que representa un total de 15 horas aproximadas de grabación. A eso se suman las interacciones y fragmentos que no han sido grabados pero que se registraron a partir de la toma de notas. La duración de las interacciones, que puede fluctuar entre un minuto y 30 minutos, varía de acuerdo con la índole del trámite o consulta.

En el procesamiento de los datos tenemos en cuenta distintas variables sociodemográficas (sexo, edad, nivel socioeducacional) y contextuales (estilos según contextos situacionales e imagen personal que se desea proyectar) (Hernández Campoy y Almeida 2005), además del tipo de institución a la que pertenece la interacción (institución pública/institución privada) y la relación existente entre los hablantes, considerando las dimensiones de poder y solidaridad propuestas por Brown y Gilman (1960).

\section{El ACTO DE HABla SALUdO Y SU PRODUCCiÓN EN ENCUENTROS DE SERVICiO DE TIPO INSTITUCIONAL}

En tanto actos de habla expresivos, los saludos adquieren particular significación «como emergentes lingüísticos de cortesía verbal» (Rigatuso 2003: 172). Constituyen actos de habla fundamentales tanto en la secuencia de apertura como en la de cierre de los intercambios. En este sentido, Goffman destaca el valor de los saludos de inicio de los intercambios comunicativos así como los de despedida y su efecto en las relaciones interpersonales:

Irving Goffman, el iniciador de la 'labor de imagen', indica que el saludo es necesario para mostrar que la relación que existía al final del último encuentro permanece inalterada, a pesar de la separación, y que la despedida es necesaria para 'resumir el efecto del encuentro sobre la relación e indicar qué es lo que cada uno de los participantes puede esperar de los demás cuando vuelvan a encontrarse' (Goffman 1955). [...] Después de haber establecido relaciones mutuas mediante el saludo, los participantes pueden pasar a concretar cualquier 'negocio' (Hudson 1981: 140).

Tal como sostienen Ferrer y Sánchez Lanza, el saludo como acto de habla universal se manifiesta de manera particular en cada cultura y se realiza mediante fórmulas muy ritualizadas que no transmiten información nueva pero conllevan «una importante carga de afectividad» (2002: 30). Además, como señala Rigatuso (2003: 172), el saludo es uno de los recursos interaccionales de manifestación de la comunión fática «cuya función primaria consiste en crear un ambiente de solidaridad que permita entablar una conversación placentera para los interlocutores»(Haverkate 1994: 57). En 
su realización «el saludo forma parte de una pareja adyacente cuyos miembros suelen ser idénticos» (Haverkate 1994: 84). Entre las funciones que Haverkate atribuye al saludo se encuentran las siguientes:

I) el saludo sirve para abrir el canal comunicativo; es decir, funciona como señal para llamar la atención del interlocutor, incitándole a participar en un intercambio verbal;

II) el saludo puede contribuir a evitar que se produzca una tensión social, provocada cuando dos personas se encuentran en una situación comunicativa potencial sin cruzar palabra; en ese caso, el saludo es el acto de habla que se presta, por excelencia, a introducir la comunión fática;

III) de acuerdo con la fórmula seleccionada, el saludo sirve para establecer o confirmar una determinada relación interaccional, tal como se define por factores como posición social, grado de intimidad y afecto (Haverkate 1994: 85).

El saludo puede adquirir diversas formas, puede darse de manera explícita o implícita (a través de sonrisas, miradas), pero generalmente la ausencia de saludo, y particularmente en situaciones de atención al público, es percibida por los usuarios como una muestra de descortesía (Placencia 2001: 195-196; Sánchez Lanza 2003: 337; Murillo Medrano 2004: 189). Así, Ferrer y Sánchez Lanza definen al saludo como un acto bidireccional que «exige ser respondido con otro saludo y si el que lo emite no obtiene respuesta, esto es percibido como un comportamiento sumamente descortés que daña la imagen positiva» (2002: 28).

En cuanto a la estructura que puede presentar este acto de habla, Haverkate realiza una distinción entre los saludos monoléxicos del tipo hola o adiós, que carecen de contenido proposicional, y los que se constituyen de dos o tres palabras, como ¿qué tal?, ¿cómo te va?, que se refieren a la vida personal del interlocutor pero se interpretan en un sentido simbólico y no se espera una respuesta. En el mismo sentido, en cuanto al repertorio de fórmulas de saludo, Ferrer y Sánchez Lanza (2002) distinguen entre las formas portadoras de significado y las carentes de él. Las primeras, que aunque han perdido su carga semántica sí pretenden una devolución del saludo, son preguntas alusivas a la salud (¿cómo estás?, ¿cómo andás?), a actividades personales (¿cómo te va?, ¿qué hacés?, ¡que te vaya bien!), y fórmulas alusivas al tiempo (buen/os dia/s, buenas tardes). Entre las formas carentes de significado incluyen formas simples como ;hola! y jadiós! Asimismo, las autoras se refieren a la acumulación de saludos de inicio y de despedida, que, como veremos en el análisis de nuestro corpus, suele ser muy frecuente en las interacciones registradas.

En el desarrollo de los encuentros de servicio la instancia de apertura del intercambio, que incluye los saludos de inicio de interacción, así como 
la de cierre, con los saludos de despedida, constituyen instancias de particular interés, ya que la índole de los saludos de inicio puede determinar el tono de la interacción que va a producirse y predisponer en algún sentido, positivo o negativo, a los participantes, mientras que los saludos de cierre pueden ser indicadores de la evaluación de los hablantes acerca del intercambio producido. Al respecto, Rigatuso destaca el valor de estas instancias en el estudio de los encuentros de servicio de tipo comercial:

Esta instancia [de apertura], junto a las secuencias correspondientes al cierre de la interacción, representan instancias medulares del discurso de los encuentros de servicio comerciales, en tanto pueden plasmar y ser índice al mismo tiempo, en el primer caso, de la índole de la relación interpersonal que se construye y se negocia entre vendedor y cliente y, en forma concomitante, del grado de formalidad y el tono que van a primar en el desarrollo de toda la interacción comercial. En el segundo caso, el del cierre, pueden incidir y ser reflejo de la percepción y evaluación del hablante respecto del tipo de interacción verificada, y, en relación con ello, de sus expectativas futuras respecto de la realización de compras en el mismo local comercial (Rigatuso 2015: 1451).

Tal como lo plantea Areiza Londoño, el saludo se produce entre personas que participan en una relación mediada por convenciones sociales donde se da un reconocimiento solidario de los sujetos (2005: 82). En el caso particular de los saludos producidos en contextos formales, el autor señala que estos se utilizan «como parte de la etiqueta sociocultural impuesta para ocasiones protocolarias, dándose así el estereotipo del acto de habla» (Ibídem). Así entendido, el saludo como acto no-sincero es caracterizado por el investigador como un comportamiento impuesto o realizado estratégicamente, pero ineludible para cualquier relación de convivencia (2005: 82-83).

En este tipo de saludos, en los que adquiere gran peso la convención social y el compromiso generado en la interacción, el otro es reconocido en su rol o en su instrumentalidad mientras se desconoce su identificación y humanidad, de manera que «el lenguaje circula protocolaria, estratégica y cortésmente por el mundo social» (Areiza Londoño y García Valencia 2003: 81). Este parece ser el tipo de saludo que predomina en los dominios institucionales que estudiamos, contextos formales en los que el otro es reconocido en su rol de empleado o usuario. Sin embargo, como veremos a continuación, en distintos puestos de atención al público del ámbito educativo estudiados, los participantes se conocen mutuamente y tienen mucha frecuencia de trato, cuestión que parece favorecer la producción de saludos más sinceros, en los que se reconoce al otro como sujeto individual y hasta se lo llama por su nombre, llegando a producirse intercambios de saludos que se asemejan a breves conversaciones. 


\subsection{El saludo en la instancia de apertura del hecho comunicativo}

Los saludos de inicio de los intercambios comunicativos tienen lugar en la instancia de apertura conversacional según la estructura de la conversación propuesta por van Dijk (1983).

Entre las fórmulas de saludo que utilizan los empleados y usuarios de estas instituciones se encuentran las siguientes, que presentamos siguiendo la clasificación propuesta por Ferrer y Sánchez Lanza (2002: 31-34):

$\rightarrow$ Formas carentes de significado:

- Lexías simples: hola.

$\rightarrow$ Formas portadoras de significado:

- Preguntas alusivas a la salud: qué tal?, cómo estás?, cómo andás?, sí(.) qué tal ? ${ }^{4}$. Estas preguntas pueden aparecer solas pero frecuentemente se registran precedidas por la forma simple hola: hola (.) qué tal?.

- Preguntas alusivas a actividades personales: hola (.) cómo te va?, cómo va? (.) bien?

- Fórmulas alusivas al tiempo: buen día, buenos días, hola (.) buen día

$\rightarrow$ Acumulación de saludos de inicio: hola (.) qué tal? buenas tardes, hola

(.) cómo estás?, buen día (.) qué tal?, hola (.) qué tal? cómo va? bien?

Resulta importante ofrecer una contextualización del modo en que se presentan los comienzos de interacción en los dominios seleccionados, ya que esto puede variar de acuerdo con la índole de la institución o el tipo de ventanilla de la que se trate, y predeterminar el modo en que se inserta el saludo. Así, en las instituciones del dominio educativo es frecuente la formación de filas de usuarios delante de los puestos de atención al público, de manera que cada usuario sabe que ha llegado su turno cuando se retira el usuario anterior, y en ese momento se acerca para ser atendido. En algunos casos, cuando se forma una única fila para esperar la atención de empleados que trabajan en diferentes escritorios, los empleados suelen realizar un llamado de atención, como parte de la instancia de preparación dentro de la estructura conversacional (van Dijk 1983), para indicarle al usuario que ha llegado su turno a través de expresiones como ¿quién sigue?, de uso frecuente también en la interacción comercial de la misma comunidad (Rigatuso 2015), sí (.) adelante o que pase el que sigue. Además de la índole de la institución en relación con el modo en que se desarrollan los comienzos de interacción en cada uno de los puestos de atención al público estudiados, existen otros factores que condicionan las distintas posibi-

\footnotetext{
${ }^{4}$ Para transcribir las citas textuales que incluimos en el cuerpo del texto seguimos las mismas normas de transcripción que empleamos en la transcripción de ejemplos.
} 
lidades en que se puede presentar la apertura conversacional, tales como la existencia o no de conocimiento previo entre empleados y usuarios y la frecuencia en el trato.

En este sentido, en los intercambios objeto de estudio resulta de interés considerar la ausencia o presencia de saludo y los esquemas en los que este se inserta en las instancias iniciales de la interacción institucional. Al respecto, el análisis de los datos muestra las siguientes posibilidades de presencia / ausencia de producción de saludos:

a) saludo recíproco,

b) forma de apertura o llamado de atención + saludo,

c) saludo + fórmula de tratamiento y

d) ausencia de fórmula de saludo.

\subsubsection{Saludo recíproco}

En las distintas interacciones que conforman nuestro corpus hemos registrado que la forma más frecuente de apertura consiste en los intercambios de saludos entre empleados y usuarios, luego de los cuales los usuarios introducen su consulta, o plantean el motivo de su presencia, tal como se observa en los siguientes fragmentos:

(1)

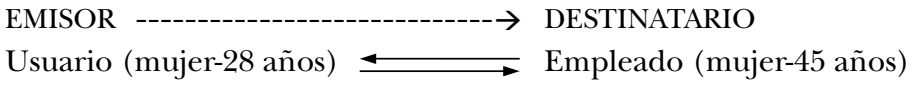

Contexto: El usuario [U] se acerca a una ventanilla de una institución educativa pública de nivel superior para retirar un certificado ${ }^{5}$ :

-[U] ho:la (.) buen dí:a ((sonriendo))

-[E] buenos días ((se levanta del escritorio y se acerca a la ventanilla))

(2) EMISOR DESTINATARIO

Usuario (mujer-30 años)

Contexto: U se dirige a una ventanilla de una institución educativa pública en la que los docentes realizan trámites para efectuar una consulta:

$-[\mathrm{U}]$ hola:

-[E] hola qué tal? cómo va (.) bien?

-[U] bie:n ((le entrega la documentación a firmar))

Nótese que en algunos casos, como sucede en este último ejemplo, se produce una acumulación de saludos que resulta más cortés que la producción de una forma simple, ya que formas de ese tipo, cuando se producen con un tono que permite interpretarlas como sinceras, parecen promover

${ }^{5}$ En las transcripciones [E] indica empleado y [U] usuario. 
un mayor acercamiento entre los interlocutores, dependiendo del grado de formalidad/informalidad del tipo de saludos acumulados. Asimismo, en el último ejemplo observamos la respuesta a la pregunta de la empleada qué tal? cómo va (.) bien? Si bien los saludos y las preguntas de este tipo se han lexicalizado, por lo que no se espera una respuesta que recupere el significado literal, en ocasiones, tal vez por el tono de sinceridad de la pregunta y por el tipo de formulación, tanto empleados como usuarios suelen responder con la forma bien, como sucede en dicho ejemplo y en los que transcribimos a continuación:

(3) EMISOR

\section{DESTINATARIO}

Empleado (mujer-30 años)

Contexto: U se acerca a una ventanilla de una institución educativa pública de nivel superior:

-[E] ((se acerca a la ventanilla con una sonrisa) $)$ hola::=

-[U] =ho::la (.) cómo estás? qué tal? ((sonriendo))

-[E] bie:n (.) vos?

-[U] bien bien

(4) EMISOR

\section{DESTINATARIO}

Usuario (mujer-31 años)

Contexto: $\mathrm{U}$ ingresa a la oficina administrativa de la universidad privada en la que se desempeña como docente:

-[U] ho:la qué tal chicas permiso

-[E] hola cómo estás claudia?

-[U] bie:n bien todo bien (.) eh te hago una consulta:

Resulta notorio en las transcripciones que la expresión bien como respuesta en boca de empleados y de usuarios suele emitirse repetida o con alargamiento vocálico (bie::n, bien bien, bie:n bien todo bien), aspectos que aumentan la expresividad de las emisiones contribuyendo a la afiliación entre los interlocutores. En este punto, es necesario recordar que el saludo es una forma inicial de conversación de contacto. Los alargamientos vocálicos, que también observamos en la producción de los saludos por parte de ambos participantes (hola: ho::la), han sido registrados también por Rigatuso en los encuentros de servicio comercial, donde connotan «un efecto de mayor cortesía, cercanía y amabilidad» (Rigatuso 2015), y es evaluado en el mismo sentido por los hablantes entrevistados por la investigadora.

\subsubsection{Forma de apertura o llamado de atención + saludo}

Según anticipamos, en algunas interacciones los empleados inician el intercambio con un llamado de atención (¿quién sigue?, que pase el que sigue) 
o utilizan una forma de apertura del tipo sí o $\underset{\iota}{s i}$ ? para indicarle al usuario que la prestación del servicio se pone en marcha (Rigatuso 2015). En estos casos, luego del llamado de atención o forma de apertura, los empleados saludan o responden al saludo del usuario.

(5)

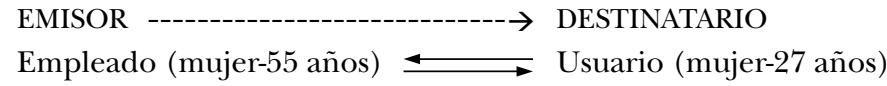

Contexto: Una empleada de una institución educativa pública de nivel superior sale de su oficina y se dirige al mostrador de atención al público:

-[E] sí:

-[U] hola buen [día (.) qué tal?]

-[E] [buen día] mh?

(6) EMISOR

DESTINATARIO

Empleado (mujer-42 años)

Contexto: U se dirige a una ventanilla de una institución educativa pública en la que los docentes realizan trámites para efectuar una consulta:

-[E] que pase el que sigue

-[U] ( (se acerca al escritorio y toma asiento $))$ hola:

-[E] cómo estás?

\subsubsection{Saludo + fórmula de tratamiento ${ }^{6}$}

Resulta frecuente en muchas de las interacciones registradas el uso del nombre de pila del interlocutor acompañando el saludo tanto en boca de empleados como de usuarios, como se observa en el fragmento que presentamos a continuación, o en el ejemplo (4) transcripto más arriba:

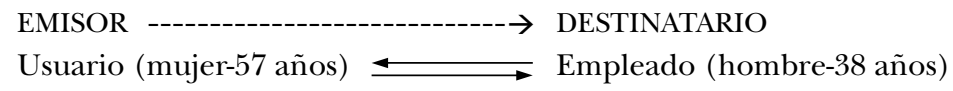

Contexto: U, docente de la institución, se acerca a una ventanilla de una institución educativa pública de nivel superior para realizar una consulta:

-[U] hola leandro (.) cómo estás?

-[E] qué tal susy?

Los usos del nombre de pila del interlocutor en el saludo constituyen una clara manifestación de cortesía y se orientan a la focalización y a la per-

${ }^{6}$ En su estudio sobre la dinámica de las fórmulas de tratamiento en instancias iniciales de la interacción comunicativa del español bonaerense, Rigatuso destaca la importancia de las fórmulas de tratamiento acompañando a un saludo en la apertura conversacional (1987: 171-174). 
sonalización del saludo ${ }^{7}$ y de la interacción en general. En relación con el tipo de relación que prefigura el uso del nombre propio del interlocutor en el saludo, Areiza Londoño señala:

El nombre propio juega un papel muy importante en las relaciones intersubjetivas, que comienzan con el acto de habla de saludar [...]. El nombre propio prefigura una relación cercana o por lo menos una relación anterior en la que ha habido un intercambio simbólico de los nombres al interior de los juegos de lenguaje en toda comunidad. Quien le da su nombre al otro le abre su mundo, le muestra parte de su intimidad y le entrega la «llave» para futuros contactos (Areiza Londoño 2005).

El uso de los nombres de pila en la producción del saludo se registra en ocasiones en algunas de las ventanillas de las instituciones educativas de nivel superior, tanto la pública como la privada, en intercambios en los que hay conocimiento previo entre los participantes y un trato más frecuente, ya sea por ser docentes de la institución o alumnos avanzados. Por el contrario, no se han registrado con mucha frecuencia estos usos en los saludos en ventanillas de la institución del ámbito educativo vinculada con la labor docente, donde en general no hay tanto conocimiento previo entre los interactuantes o no se conocen por su nombre, por lo cual los saludos suelen ser más despersonalizados.

En relación con estas posibilidades, es importante destacar el valor de la presencia del nombre de pila frente a su ausencia en interacciones institucionales en las que suele prevalecer impersonalidad. En tal sentido, el nombre de pila acompañando los saludos constituye uno de los usos que orientan la interacción institucional hacia un estilo comunicativo que plantea una relación «más personal y menos institucionalizada» (Prego Vázquez 2007: 119), promoviendo la afiliación (Bravo 2003) y el acercamiento entre empleados y usuarios. Esto se relaciona con el tipo de vínculo construido entre los participantes. Así, en su estudio sobre la interacción comercial en Quito, Placencia refiere al uso del nombre de pila en los saludos como marca de una relación amigable y no anónima. Al respecto señala:

The use of first names, in fact, signals that the participants are not simply acting in their institutional roles as 'anyones' (any anonymous shopkeeper and customer), but as specific individuals or 'someones'. In other cases, if first names are absent, it may be other elements of the opening, such as howare-you enquiries, that enact the personal and friendly frame that one or both participants propose to adopt for the encounter (Placencia 2004: 222).

${ }^{7}$ Según los estudios de Rigatuso, la focalización del destinatario y la personalización del mensaje, así como la contribución a la construcción de la identidad de cliente, constituyen funciones pragmáticas relevantes de las fórmulas de tratamiento en español bonaerense (Rigatuso 2000 y 2011a). 
En la misma línea, Rigatuso examina el uso del nombre de pila acompañando los saludos en las secuencias de apertura y cierre de la interacción comercial en español bonaerense, considerando su función en la personalización de la interacción y en la construcción de la identidad de cliente (Rigatuso 2011a).

Tal como relevamos en nuestro corpus, en este uso resulta determinante la variable referida al tipo de relación existente entre los participantes, ya que el conocimiento previo entre empleados y usuarios constituye un factor indispensable para la elección del nombre de pila o sobrenombre del destinatario, especialmente en la instancia de apertura. Generalmente se registra en boca de empleados jóvenes o de edad mediana (aproximadamente en una franja etaria que comprende desde los 25 años hasta los 50 años) al dirigirse a alumnos o docentes de distintas edades que son usuarios habituales de los puestos de atención al público estudiados. En el caso de los usuarios, cuando existe ese conocimiento previo y trato cotidiano, la elección del nombre de pila en el saludo es también lo habitual (Julián 2017).

\subsubsection{Ausencia de fórmula de saludo}

En el corpus de análisis observamos interacciones en las que los empleados no saludan ni retribuyen el saludo del usuario. Esta ausencia del saludo en las instancias iniciales se encuentra en directa vinculación con las estrategias de comienzo del intercambio y tiene grados diferentes de implicación en relación a la (des)cortesía.

En algunas de dichas oportunidades hemos registrado el inicio de las interacciones por parte de los empleados a través de las formas de apertura ya referidas, como sí, decime, sí (.) adelante o sí(.) quién sigue?, luego de lo cual con frecuencia, aunque no siempre, el usuario produce un saludo e inmediatamente introduce su consulta.

EMISOR $\rightarrow$ DESTINATARIO

Empleado (hombre-50 años) $\longleftrightarrow$ Usuario (mujer-40 años)

Contexto: U se dirige a una ventanilla de una institución educativa pública en la que los docentes realizan trámites para realizar una consulta:

-[E] quién sigue?

-[U] hola (.) buen día (.) quería saber cómo tengo que hacer para ((plantea la inquietud))

-[E] tenés que completar el formulario ((continúa dando las indicaciones))

Otras interacciones en las que también registramos la ausencia de respuesta del empleado al saludo del usuario ocurre cuando los primeros utilizan fórmulas de tratamiento como ¿niña?, ¿muchacho?, ¿chicas-os? con 
entonación ascendente de pregunta en la instancia de apertura conversacional (Rigatuso 1987). Dichas formas pueden aparecer en reemplazo del saludo y, tal como se da también en la interacción comercial, sustituyen a la expresión ¿̇qué necesita/necesitás? (Rigatuso 1987, 2000 y 2015). El empleo elíptico de una fórmula constituye una de las funciones características de los tratamientos en la instancia de apertura conversacional del español bonaerense y resulta, en consecuencia, una práctica a la que el usuario está acostumbrado por su uso en distintos ámbitos de prestación de servicios de la comunidad (Ibídem). A continuación transcribimos algunos ejemplos de tales usos:

(9) EMISOR --------------- $\rightarrow$ DESTINATARIO

Empleado (hombre-35 años) $\longleftrightarrow$ Usuario (hombre-28 años)

Contexto: U se presenta en una ventanilla de una institución educativa pública de nivel superior. $\mathrm{E}$ termina de atender a otro usuario y se dirige a U:

-[E] muchacho?

-[U] qué tal? tengo que entregar estos papeles

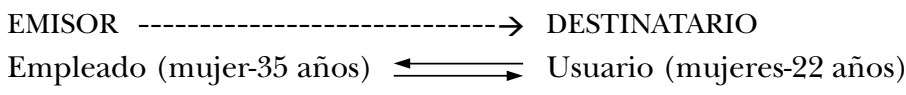

Contexto: Las usuarias se acercan a una ventanilla de una institución educativa privada de nivel superior de la que son alumnas. La empleada las recibe:

$-[\mathrm{E}]$ chicasss?

-[U] hola (.) tenemos una duda...

Asimismo, hemos registrado usos similares con la forma pronominal ¿vos?, como se observa en el siguiente fragmento de interacción:

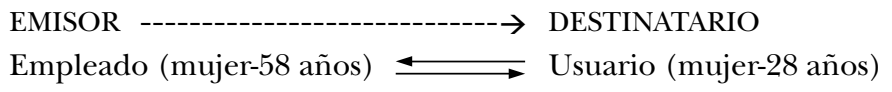

Contexto: U se presenta en una ventanilla de una institución pública en la que los docentes realizan trámites para consultar a la empleada por las fechas de la inscripción en un listado. E termina de atender a otra usuaria y dirige su mirada a $\mathrm{U}$ :

$-[\mathrm{E}] \operatorname{vos}$ ?

-[U] hola (.) quería hacer una consulta (.) para inscribirme en el listado (.) hasta cuándo hay tiempo?

-[E] hasta el trece de julio

En otras interacciones, en cambio, en la instancia de apertura se produce el contacto visual del empleado acompañado de silencio (Rigatuso 2015) para indicar al usuario que puede introducir su consulta. En los siguien- 
tes ejemplos el empleado mira al usuario e ignora su saludo, rompiendo con el par de adyacencia:

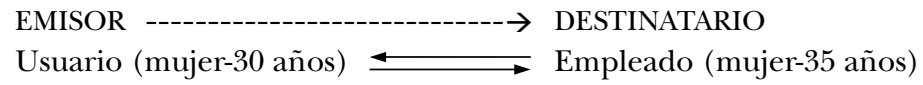

Contexto: $\mathrm{U}$, docente de la institución, ingresa a la secretaría de una institución educativa privada de nivel superior para realizar una consulta:

-[U] ho:la

-[E] ((no saluda, sigue trabajando en la computadora por unos segundos hasta que se da vuelta y mira a U))

-[U] disculpame (.) te hago una consulta (.) ya terminamos de tomar el final (.) tenemos que firmar algo ahora o cuando traemos las notas?

-[E] sí (.) el cuaderno ((le muestra dónde firmar))

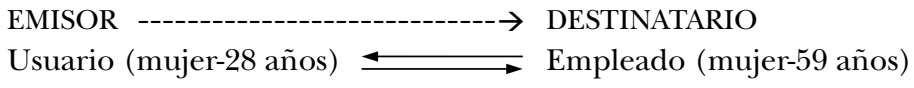

Contexto: U se dirige a una ventanilla de una institución educativa pública en la que los docentes realizan trámites para certificar documentación. E la mira:

-[U] hola: ((le entrega la documentación))

-[E] bueno (.) documento?

Estos últimos constituyen los casos de ausencia de saludo más vinculados a las prácticas descorteses. Tal como relevamos a partir de los tests de hábitos sociales, la presencia/ausencia de saludos o respuesta a los saludos por parte de los empleados constituye un factor muy importante para los usuarios en la definición de la atención como cortés o descortés. En tal sentido, en consonancia con otras investigaciones sobre (des)cortesía en instituciones en otras variedades de español (por ejemplo, Placencia 2001; Murillo Medrano 2004), los usuarios consideran que el saludo constituye un acto de habla cortés fundamental en la interacción entre empleados y usuarios, y en reiteradas oportunidades aluden a la ausencia de saludos por parte de los empleados al referirse a los elementos lingüísticos codificadores de descortesía en la atención al público en las instituciones que nos ocupan.

De acuerdo con lo registrado en nuestro corpus, hemos notado una mayor tendencia a que los usuarios de las instituciones del ámbito educativo estudiadas realicen el primer movimiento hacia la cortesía en cuanto a los saludos de inicio de interacción. En estudios previos sobre la misma comunidad, Rigatuso encuentra una tendencia similar en la interacción comercial, en la que son generalmente los compradores quienes realizan la contribución inicial a la cortesía a través del saludo al llegar a la caja (Rigatuso 2003: 173). Asimismo, en nuestro estudio sobre la interacción en instituciones del ámbito económico en español bonaerense hemos registrado la misma tendencia (Julián 2012). 


\subsection{El saludo en la instancia de cierre del hecho comunicativo}

Como hemos señalado, el saludo en la instancia de cierre de las interacciones puede funcionar como indicador de la evaluación de los hablantes acerca del intercambio producido y predisponer en algún sentido para futuros encuentros.

Los saludos de despedida registrados en las instituciones del ámbito de la educación que nos ocupan suelen estar precedidos por una presecuencia de cierre que se da en la instancia de conclusión de la estructura de la conversación (van Dijk 1983), producida por empleados y/o usuarios, que consiste en expresiones que preparan el cierre de la interacción, tales como bien, muy bien, bueno, listo, bárbaro, perfecto, seguidas generalmente del agradecimiento por parte del usuario y la respuesta del empleado. A continuación se insertan los saludos de despedida, actos de habla que constituyen la instancia de terminación, es decir, el fin de la conversación.

En lo relativo a las variantes del saludo, en la instancia de cierre se registra el uso de las siguientes fórmulas de saludo por parte de ambos participantes, presentadas, como en el caso de los saludos de la instancia de apertura, de acuerdo con la clasificación de Ferrer y Sánchez Lanza (2002):

$\rightarrow$ Formas carentes de significado:

- Lexías simples: chau, adiós.

$\rightarrow$ Formas portadoras de significado:

- Formas alusivas a actividades personales: Ubicamos aquí algunas expresiones de buenos deseos acompañando el saludo: suerte!, hasta luego (.) que tengan buen viaje!, felices vacaciones! y felices fiestas!, que descanses mucho mañana eh?

- Fórmulas alusivas al tiempo: hasta luego, nos vemos.

$\rightarrow$ Acumulación de saludos de cierre: chau (.) hasta luego, nos vemos (.) hasta luego! Circunstancialmente aparecen acumuladas, a modo de saludos de cierre, expresiones de buenos deseos como nos vemos! felices fiestas! felices vacaciones!, felices vacaciones! y felices fiestas!, hasta luego (.) que tengan buen viaje!

Los usuarios emiten un saludo de despedida en la mayoría de las interacciones, aunque no siempre reciben la respuesta a su saludo. En ocasiones el agradecimiento del usuario puede reemplazar el saludo y operar como forma de cierre que equivale al saludo de despedida, o bien la respuesta del empleado al agradecimiento del usuario puede sustituir su saludo de despedida, comportamiento que resulta muy frecuente en las interacciones estudiadas. Asimismo, cuando el usuario es derivado a otro sector o ventanilla de la institución puede simplemente agradecer sin emitir un saludo de despedida. 
A continuación ofrecemos ejemplos que ilustran aquellos cierres de interacción que resultan más frecuentes en las instituciones del ámbito educativo seleccionadas. Los clasificamos según sean:

a) saludo recíproco,

b) saludo + fórmula de tratamiento,

c) ausencia de fórmulas de saludo del usuario y su reemplazo por fórmulas de agradecimiento,

d) ausencia de saludo por parte del empleado y su reemplazo por la respuesta al agradecimiento o la expresión de buenos deseos y

e) ausencia de saludo por parte de ambos participantes.

\subsubsection{Saludo recíproco}

En primer lugar ejemplificamos aquellos casos, relevados con mucha frecuencia, en los que ambos participantes saludan o responden al saludo de despedida:

(14) EMISOR $\rightarrow$ DESTINATARIO

Usuario (mujer-27 años)

Contexto: U realiza un trámite en una institución educativa pública de nivel superior. Al finalizar el intercambio los participantes se despiden:

-[U] bueno (.) muy amable

$-[\mathrm{E}]$ listo

-[U] [hasta luego]

$-[\mathrm{E}]$ [hasta luego]

(15)

EMISOR ---------------------- $\rightarrow$ DESTINATARIO

Usuario (mujer-25 años)

Contexto: U realiza una consulta en una institución educativa pública de nivel superior, planteando que no podrá estar presente en la fecha de concreción del trámite porque realizará un viaje con su madre. Luego de recibir las indicaciones necesarias las participantes se despiden:

$-[\mathrm{U}]$ bueno

-[E] bueno (.) bárbaro (.) hasta luego que tengan buen viaje!

-[U] chau hasta luego

(16) EMISOR

\section{DESTINATARIO}

Usuario (mujer-30 años)

Contexto: U realiza una consulta en una institución educativa privada de nivel superior en la que se desempeña como docente. Al concluir se despide: 
-[U] bueno muchas gracias chicas

-[E] no de nada

-[U] felices vacaciones! y felices [fiestas]

-[E] [igualmente] (.) chau hasta luego

Es importante destacar la tendencia a la personalización que se registra en algunos de los saludos de los fragmentos anteriores y en otros fragmentos de nuestro corpus, tendencia que se concreta a través de la expresión de buenos deseos (felices vacaciones!, felices fiestas!, que tengan buen viaje!, suerte!).

\subsubsection{Saludo + fórmula de tratamiento}

Tal como registramos en la instancia de apertura, en la instancia de cierre hemos relevado también el uso del nombre de pila del destinatario acompañando el saludo de despedida, tanto en boca de empleados como de usuarios. Justamente, el único uso de fórmulas de tratamiento en el saludo de despedida que aparece en nuestro corpus corresponde al nombre de pila. Los siguientes ejemplos ilustran este empleo:

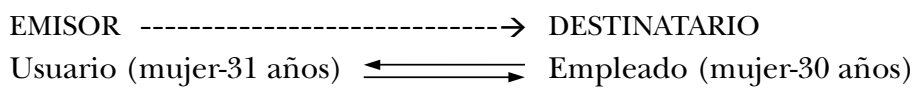
da de nivel superior en la que se desempeña como docente. Al concluir se despide:

-[U] bue:no (.) listo (.) nos vemos!

-[E] nos vemos Valeria

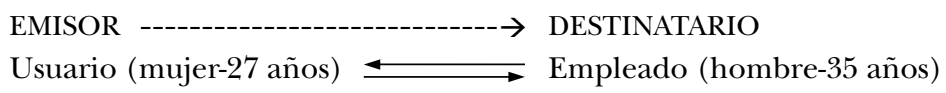
nivel superior en la que realiza sus estudios. Al finalizar la misma los participantes se despiden:

-[U] bue:no:: bárbaro buenísimo (.) gracias ariel [hasta luego]

-[E] [de nada $\left.{ }^{\circ} \mathrm{chau}^{\circ}\right]$

Tales usos se registran especialmente en algunos de los puestos de atención de las instituciones educativas de nivel superior, en las que resulta más frecuente la existencia de conocimiento previo entre empleados y usuarios, sean docentes o alumnos avanzados o de carreras de posgrado, por su frecuencia de trato durante las consultas y trámites, a lo que se suma, en ocasiones, los contactos a través del correo electrónico entre empleados administrativos y usuarios por solicitudes de datos, documentación o informes. 
2.2.3 Ausencia de fórmulas de saludo del usuario y su reemplazo por fórmulas de agradecimiento

Como señalamos más arriba, en ocasiones ocurre que los usuarios no emiten saludo de despedida y lo reemplazan por un agradecimiento. Una de las posibilidades en que se verifica esta situación es en aquellos casos en que no abandonan la institución sino que han sido derivados a otra ventanilla o a otro sector del establecimiento, o bien por tener que desplazarse un momento para realizar otro trámite o una fotocopia. En esos casos emplean, como forma de cierre de la interacción, el acto de agradecimiento, como ilustra el siguiente ejemplo:

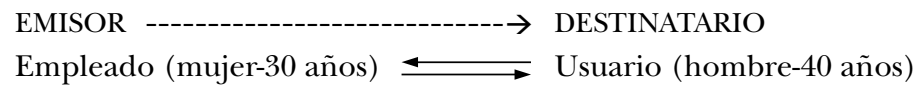

Contexto: U realiza una consulta en una institución educativa pública de nivel superior. En las instancias finales de la interacción, E le indica que debe dirigirse a otro sector para solicitar la documentación requerida para completar el trámite:

-[E] tenés que traerme el analítico para que veamos las materias en qué: fecha las rendiste (.) para pode:r [sacar los programas]

-[U] [bue:no:] (.) ahora voy allá: (.) gracias

-[E] de nada

Como se observa a partir del ejemplo transcripto, la ausencia de saludos está debidamente justificada por la situación, por lo que probablemente no genere incomodidad en los interlocutores. Además, se produce el agradecimiento como forma de cierre.

Por otra parte, en los casos en que los usuarios solicitan en ventanillas u oficinas información acerca de la ubicación de algún sector de la institución, suelen recibir la respuesta y agradecer sin producir un saludo de despedida. En esas oportunidades, los empleados suelen no responder al agradecimiento ni saludar, otorgando prioridad a la celeridad y al aspecto transaccional de la interacción.

\subsubsection{Ausencia de saludo por parte del empleado y su reemplazo} por la respuesta al agradecimiento o la expresión de buenos deseos

Asimismo, en otros intercambios los empleados no responden a los saludos de despedida del usuario pero finalizan la interacción respondiendo al agradecimiento, expresando buenos deseos o, lo que resulta poco frecuente, produciendo un agradecimiento, que funcionaría en esos casos como despedida, por lo que no resulta incómoda la falta de respuesta al 
saludo. Estos cierres de interacción resultan muy frecuentes en las interacciones desarrolladas en estos contextos institucionales. A continuación transcribimos un fragmento que ilustra tales situaciones:

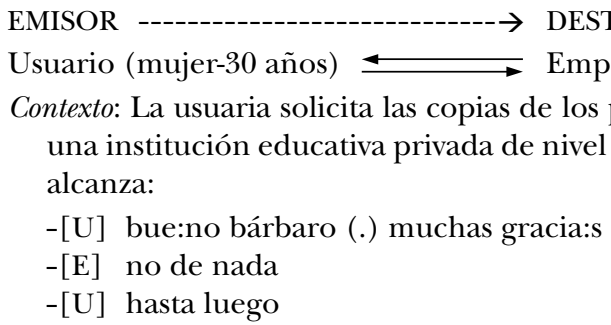

\subsubsection{Ausencia de saludo por parte de ambos participantes}

A diferencia de las situaciones anteriores, en las que la producción de un saludo de despedida se reemplaza por otra forma de cortesía, como el agradecimiento o la respuesta a este acto de habla, en otras oportunidades hemos registrado la ausencia injustificada de respuesta a los saludos del usuario por parte del empleado, como ocurre en el siguiente ejemplo:

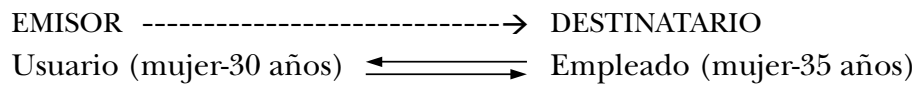

Contexto: $\mathrm{U}$ realiza una consulta en una institución educativa privada de nivel superior en la que se desempeña como docente. Al concluir se despide:

-[U] bue:no (.) bárbaro (.) muchas gracias (.) hasta luego ((E no responde y sigue trabajando))

En tales casos los empleados rompen con el par adyacente que suponen los saludos (Haverkate 1994) al no producir la respuesta esperada. Esta ausencia de respuesta a los saludos de despedida emitidos por los usuarios resulta injustificada, por lo que comportamientos de este tipo son calificados como descorteses de acuerdo con las percepciones que los usuarios tienen al respecto. En la teoría de los actos de habla, estos casos en los que no se cumple con la segunda parte del par adyacente se incluyen en la categoría de respuestas despreferidas:

Las segundas partes que satisfacen las expectativas de las primeras son denominadas respuestas preferidas en el sentido de no marcación-concepto desarrollado por los lingüistas de la Escuela de Praga-, mientras que las respuestas que implican rechazo, no aceptación, no contestación o contestación no esperada son las despreferidas o marcadas (Ferrer y Sánchez Lanza 2002: 13). 
Finalmente, hemos relevado algunos casos en los que los usuarios no saludan en la instancia de cierre de la interacción debido al fracaso de la interacción y a su evaluación negativa acerca de la atención recibida. En esas interacciones conflictivas, los empleados tampoco producen un saludo de despedida. A continuación transcribimos el final de una interacción conflictiva:

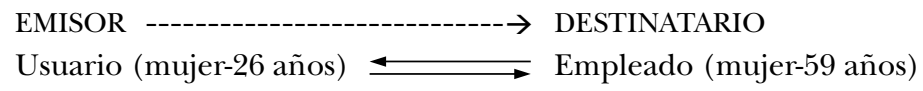

Contexto: La empleada de una institución pública del ámbito educativo en la que los docentes realizan diversos trámites interrumpe la fila por estar próximos al horario de cierre. La usuaria y su acompañante llegan a la fila justo cuando la empleada la interrumpe, por lo que deciden ir a preguntarle si pueden hacer la fila porque llegaban en ese momento y U no podría volver otro día por su trabajo. Luego de una interacción conflictiva, en la que la empleada se muestra descortés, finaliza el intercambio:

-[E] MANDÁ A A:LGUIEN CORAZÓN (.) ((sin mirarla)) PODÉS MANDAR A CUALQUIER PERSONA MI VIDA: ((tono soberbio y desafiante)) [eh?]

-[U] [bue:no::] ((Se retira enojada. Ninguno de los participantes emite un saludo de despedida))

En una mirada de conjunto, el análisis del corpus ha puesto de manifiesto la tendencia de los usuarios a manifestar cortesía en el cierre de los intercambios, especialmente en la producción del saludo de despedida, además del agradecimiento esperable en estos dominios, a diferencia de los empleados, cuya contribución a la cortesía en esta instancia suele restringirse a responder a las expresiones de cortesía de los usuarios. Asimismo, por una cuestión proxémica y de práctica etnográfica podría explicarse que el participante que saluda primero es el usuario porque es quien se retira del puesto de atención o de la institución.

\section{Conclusiones}

El estudio de la dinámica del saludo en las instituciones del dominio educativo seleccionadas, correspondientes a la variedad del español bonaerense, permitió observar que los saludos se orientan hacia el reconocimiento del otro y contribuyen a la creación de un ambiente de solidaridad y acercamiento entre los interlocutores. Cuando dicho reconocimiento no se produce, tal como sucede en aquellos casos en los que los empleados no saludan al usuario o no responden a su saludo, se produce una tensión social que deriva en una percepción negativa de dicho comportamiento, 
que resulta calificado como descortés por los usuarios. La ausencia de la producción del saludo o la producción de un acto de habla despreferido en relación al saludo emitido por el usuario emerge así como práctica descortés.

La importancia de los saludos en la comunicación entre empleados y usuarios en encuentros de servicio de esta índole radica en que la forma de apertura de las interacciones puede condicionar el tono de las mismas y el modo en que se van a desarrollar, así como la instancia de cierre puede ser reflejo de la evaluación de los hablantes acerca del intercambio producido y tener incidencia en las expectativas para futuros intercambios en esos dominios (Rigatuso 2015). En este sentido, la presencia/ausencia de saludo y el modo en que este acto de habla se produce puede reflejar o incidir en el éxito o el fracaso de la interacción en encuentros de servicio de la índole de los abordados.

A lo largo del trabajo hemos examinado la presencia/ausencia de saludo en relación con las prácticas (des) corteses en el discurso institucional, y los distintos esquemas en los que se inserta, atendiendo a las situaciones particulares en las que este acto de habla puede ser sustituido por otro, como el agradecimiento en la instancia de cierre, o por la expresión de una fórmula de tratamiento con entonación ascendente en la instancia de apertura, sin que esto conlleve consecuencias negativas en cuanto a la expresión de cortesía.

De acuerdo con nuestro análisis, algunos de los esquemas de saludo relevados tienen mayor vinculación con la expresión de cortesía y la gestión de los vínculos, y, en consecuencia, contribuyen al éxito de la interacción. Tal es el caso, por ejemplo, del uso recíproco de saludos o el uso de saludos + fórmula de tratamiento. Por el contrario, la ausencia de fórmula de saludo sin ser reemplazado por otra expresión de cortesía o sin que la situación lo justifique, como en los casos en que los usuarios no son saludados porque son derivados a otra ventanilla y no abandonan la institución, parece vincularse en mayor medida con la descortesía y con situaciones de fracaso en los encuentros de servicio de tipo institucional.

El análisis ha puesto de manifiesto que existe una mayor tendencia a que los usuarios realicen el primer movimiento hacia la cortesía en cuanto a los saludos de inicio y cierre de interacción, en consonancia con estudios previos en los que se ha registrado una tendencia similar (Rigatuso 2003; Julián 2012). En cuanto al funcionamiento de las variables extralingüísticas, la variable sexo no ha condicionado variaciones significativas en la producción de los saludos. Tampoco registramos diferencias en cuanto a la variable institución pública/institución privada. La variable edad, por el contrario, ha resultado operativa en el análisis en lo que respecta al uso del nombre de pila acompañando la emisión de saludos. 
De acuerdo con lo expuesto a lo largo de este artículo, resulta interesante destacar las diversas formas de personalización de los saludos. En tal sentido, hemos registrado, en aquellas interacciones en las que existe conocimiento previo entre los participantes y frecuencia de trato, el uso del nombre de pila o sobrenombre del usuario en boca de los empleados como modo de personalizar los saludos tanto de inicio de interacción como de despedida, así como también los agradecimientos. Tales usos se registran asimismo en boca de los usuarios al dirigirse a los empleados, y se dan tanto en vínculos simétricos como asimétricos. Si confrontamos los distintos contextos estudiados, los contextos universitarios ponen de manifiesto que, en conversaciones específicas, aparece el nombre de pila en los saludos en las dos direcciones de la díada, lo que contribuye a la construcción y mantenimiento de un vínculo interpersonal más cercano.

Además, hemos registrado otras formas de personalización de estos actos de habla tales como, en el inicio, las preguntas del tipo qué tal? cómo $v a$ ? y sus respuestas, o, en la despedida, las expresiones de buenos deseos. $\mathrm{Al}$ respecto, nos interesa señalar la importancia que adquiere en estos contextos la focalización del destinatario y la personalización del mensaje, ya que se trata de valores que, por su frecuencia de uso en otros dominios de interacción, forman parte del comportamiento y de la competencia comunicativa de los hablantes de la comunidad (cfr. Rigatuso 2000 y 2011b). 


\section{BIBLIOGRAFÍA}

AREIZA LondoÑo, Rafael (2005): «iPrimero se saluda! Una mirada sociolingüística al saludo en el Eje Cafetero, Colombia». En Jorge Murillo (ed.), Actas del II Coloquio Internacional del Programa EDICE, Estocolmo-Costa Rica, Programa Edice-Universidad de Costa Rica, 81-95.

Areiza Londoño, Rafael y Alejandro García Valencia (2003): «¿Qué significa saludar?». En Diana Bravo (ed.), Actas del Primer coloquio del programa EDICE, Estocolmo: Universidad de Estocolmo, 71-85.

Bravo, Diana (1999): «¿Imagen 'positiva' vs. Imagen 'negativa’? Pragmática sociocultural y componentes de face», Oralia. Análisis del Discurso Oral 2, 155-184.

- (2003): «Actividades de cortesía, imagen social y contextos socioculturales: una introducción». En Diana Bravo (ed.), Actas del Primer coloquio del programa EDICE, Estocolmo: Universidad de Estocolmo, 98-108.

- (2009): «Pragmática, sociopragmática y pragmática sociocultural del discurso de la cortesía. Una introducción». En Diana Bravo, Nieves Hernández Flores y Ariel Cordisco (eds.), Aportes Pragmáticos, sociopragmáticos y socioculturales a los estudios de la cortesía en español, Estocolmo - Buenos Aires: Dunken, 31-68.

Bravo, Diana y Antonio Briz (eds.) (2004): Pragmática sociocultural. Estudios sobre cortesía en español, Barcelona: Ariel.

Brown, Roger y Albert Gilman (1960): «The pronouns of power and solidarity». En Thomas Sebeok (ed.), Style in Language, New York: Massachusetts Institute of Technology, 253-275.

Drew, Paul y Marja-Leena Sorjonen (2000): «Diálogo institucional». En Teun van Dijk (comp.), El discurso como interacción social. Estudios sobre el discurso II. Una introducción multidisciplinaria, Barcelona: Gedisa, 141-178.

Duranti, Alejandro (2000): Antropología lingüistica, Madrid: Cambridge University Press.

Ferrer, María Cristina y Carmen SÁNChez LANZA (2002): Interacción verbal. Los actos de habla, Rosario: UNR Editora.

Gabbiani, Beatriz e Irene Madfes (org.) (2006): Conversación y Poder. Análisis de interacciones en aulas y consultorios, Universidad de la República, Montevideo: Fondo Clemente Estable.

GOFFMAN, Ervin (1967): Interaction ritual. Essays on face to face behaviour, New York: Doubleday Anchor Books.

GuMPERZ, John (ed.) (1982a): Discourse strategies, New York: Cambridge University Press.

- (1982b): Language and social identity, New York: Cambridge University Press.

- (2001): «Interactional Sociolinguistics: A Personal Perspective». En Deborah Schiffrin, Deborah Tannen y Heidi Hamilton (eds.), The Handbook of Discourse Analysis, Malden, MA: Blackwell, 215-228. 
GuMPERZ, John y Dell HyMes (eds.) (1972): Directions in sociolingüistics. The ethnography of communication, New York: Holt, Rinehart and Winston, Inc.

HAVERKATE, Henk (1994): La cortesía verbal. Estudio pragmalingüístico, Madrid: Gredos.

Hernández Campoy, Juan Manuel y Manuel Almeida (2005): Metodología de la investigación sociolingüistica, Málaga: Comares.

Hudson, Richard (1981): La sociolingüistica, Barcelona: Anagrama.

Julián, Gisele (2012): Expresión y percepción de la (des)cortesía en puestos de atención al público en instituciones bahienses de carácter económico, Bahía Blanca: Departamento de Humanidades, Universidad Nacional del Sur.

- (2015): «(Des)cortesía verbal y puestos de atención al público en español bonaerense. Aplicación de un test de hábitos sociales». En Alicia Carrizo y Carolina Tosi (coord.), Actas del VII Coloquio Argentino de la Asociación Latinoamericana de Estudios del Discurso. Estudios del Discurso en Latinoamérica, Buenos Aires: Editorial de la Facultad de Filosofía y Letras, Universidad de Buenos Aires, 99-107.

- (2017): «Usos del nombre de pila en puestos de atención al público en español bonaerense». En Roberto Bein y Elizabeth M. Rigatuso (eds.), Asuntos de Sociolingüistica y Análisis del Discurso. Bahía Blanca: Editorial de la Universidad Nacional del Sur, Ediuns y SAEL, 57-68.

KAUL, Silvia (2008): «Tipología del comportamiento verbal descortés en español». En Antonio Briz et al. (eds.), Actas del III Coloquio Internacional del Programa EDICE, Valencia: Universidad de Valencia, 254-266.

Labov, William (1970): "The study of language in its social context», Studium Generale 23, 30-87.

López Morales, Humberto (1994): Métodos de investigación lingüistica, Salamanca: Editorial del Colegio de España.

Moreno Fernández, Francisco (1990): Metodología sociolingüística, Madrid: Gredos.

Murillo Medrano, Jorge (2004): «La cortesía verbal en Costa Rica. Percepciones de los hablantes sobre la (des) cortesía en puestos de atención al público». En Diana Bravo y Antonio Briz (eds.), Pragmática sociocultural. Estudios sobre cortesía en español, Barcelona: Ariel, 76-88.

Placencia, María Elena (2001): «Percepciones y manifestaciones de la (des)cortesía en la atención al público: el caso de una institución pública ecuatoriana», Oralia 4, 213-241.

- (2004): «Rapport-building activities in corner shop interactions», Journal of Sociolingüistics 8/2, 215-245.

- (2008): «'Hola María': racismo y discriminación en la interacción interétnica cotidiana en Quito», Discurso \& Sociedad 2/3, 573-608.

Placencia, María Elena y Diana Bravo (eds.) (2009): Actos de habla y cortesía en español, Múnich: Lincom.

Prego VÁzquez, Gabriela (2007): «Los recursos interaccionales del poder como mecanismos de negociación de identidades profesionales», Revista Internacional de Lingüistica Iberoamericana V, 1/9, 111-124. 
Rigatuso, Elizabeth M. (1987): «Dinámica de los tratamientos en la interacción verbal: preparación y apertura conversacionales», Anuario de Lingüistica Hispánica III, Valladolid: Universidad de Valladolid, 161-182.

- (2000): «'Señora (...) ¿No tenés más chico?’ Un aspecto de la pragmática de las fórmulas de tratamiento en español bonaerense», Revista Argentina de Lingüistica 16, 293-344.

- (2003): «Cortesía, tratamientos e identidad cultural en encuentros de servicio en español bonaerense». En María del Carmen Vaquero y Mabel Cernadas de Bulnes (eds.), Actas de las II Jornadas Interdisciplinarias del Sudoeste Bonaerense, Bahía Blanca, Archivo de la Memoria: UNS, 157-179.

- (2008): «¿Qué! ¿tienen calor?’. Conversación de contacto en español bonaerense: de interacciones institucionales, de servicio y sociales», Oralia 11, 133-168.

- (2011a): «Conversación de contacto y variación situacional: la construcción de identidad en dos dominios interaccionales del español bonaerense actual». En Carmen García y María Elena Placencia (dirs.), Estudios de variación pragmática en español, Buenos Aires: Editorial Dunken, 243-275.

- (2011b): Funciones sociolingüísticas y pragmáticas de las fórmulas de tratamiento en español: consideraciones sobre la variedad bonaerense, Conferencia presentada en el XIV Congresso Brasileiro de Professores de Espanhol, Niteroi, Brasil.

- (2015): «Interacción, variación y cambio: estrategias comunicativas en la interacción comercial del español bonaerense actual». En Salvio Martín Menéndez (coord.), Actas de las II Jornadas Internacionales Beatriz Lavandera: sociolingüistica y análisis del discurso, Buenos Aires: Editorial de la FFyL UBA, 1437-1470.

SÁnCHEZ LANZA, Carmen (2003): «El discurso de la cortesía en puestos de atención al público en la Argentina (instituciones de salud pública y bienestar social)». En Diana Bravo (ed.), Actas del Primer coloquio del programa EDICE, Estocolmo: Universidad de Estocolmo, 332-345.

Spencer-OAtey, Helen (2008): Culturally Speaking. Managing Rapport through Talk Across Cultures, Londres: Continuum.

Tannen, Deborah (1985): Conversational style. Analizing talk among friends, New Jersey: Ablex publishing corporation.

- (1996): Género y discurso, Barcelona: Paidós.

- (2004): «Interactional Sociolinguistics». En Ulrich Ammon et al. (eds.), Sociolinguistics: An International Handbook, Berlín: Walter de Gruyter, 181-195.

VAN Dijk, Teun (1983): La ciencia del texto, Buenos Aires: Paidós.

- (comp.) (2000): El discurso como interacción social. Estudios sobre el discurso II. Una introducción multidisciplinaria, Barcelona: Gedisa. 